\title{
Treatment and Outcome of Patients with Uterine Carcinosarcoma in a Comprehensive Cancer Network
}

\author{
K. Hapsari ${ }^{1,7}$. C. Bhugwandass ${ }^{1,6}$. G. W. J. van Rijn ${ }^{1}$ - A. A. M. van der Wurff' ${ }^{2}$ M. van 't Veer ${ }^{1} \cdot$

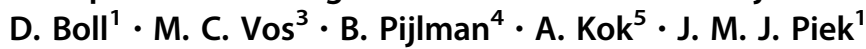

Received: 17 September 2019/Accepted: 7 December 2019/Published online: 16 December 2019

(C) The Author(s) 2019

\begin{abstract}
Aim To determine clinical characteristics, treatment modalities and survival of uterine carcinosarcoma (UCS).

Methods Data on treatment of UCS patients in the Comprehensive Cancer Network south region in the Netherlands between 2004 and 2014 were retrospectively evaluated.

Results Data of 62 patients with UCS were retrieved. Mean age at diagnosis was 69.2 years (45-95 years). Data of six patients were excluded because they did not receive any treatment. Of the 56 patients included in this study, 57.1\% presented with early-stage (FIGO I-II) disease and 42.9\% with late-stage (FIGO III-IV) disease. 46.9\% of the patients with FIGO early-stage disease received only surgical treatment, whereas $9.4 \%$ received adjuvant chemotherapy and $43.8 \%$ received adjuvant radiotherapy. Median DFS in patients with early-stage disease was 47.0 months (17.5-72.0). Adjuvant therapy did not seem to alter prognosis $(p=0.261) .16 .7 \%$ of the patients with late-stage disease received only surgical treatment, $12.5 \%$ received only chemotherapy, whereas 50\% received adjuvant chemotherapy and 20.8\% adjuvant radiotherapy after surgery. Median DFS in late-stage disease was 8.5 months (2.5-23.5). Adjuvant therapy did not seem to alter prognosis $(p=0.30)$.

Conclusion UCS with both FIGO stages I-II and III-IV has a dismal prognosis. The addition of adjuvant treatment did not seem to increase survival.
\end{abstract}

Keywords Uterine carcinosarcoma · Uterine neoplasm · Adjuvant therapy · Adjuvant chemotherapy · MMMT

\section{Introduction}

Uterine carcinosarcoma (UCS), also known as malignant mixed Müllerian tumor (MMMT), is a rare, aggressive gynecologic malignancy. It comprises less than $5 \%$ of all gynecologic malignancies, but contributes to $15 \%$ of deaths caused by gynecologic malignancies [1]. Five-year survival

K. Hapsari

kartika.hapsari@radboudumc.nl

1 Department of Obstetrics and Gynaecology, Catharina Hospital, Eindhoven, The Netherlands

2 Department of Pathology, Elisabeth-TweeSteden Hospital, Tilburg, The Netherlands

3 Department of Obstetrics and Gynaecology, ElisabethTweesteden Hospital, Tilburg, The Netherlands

4 Department of Obstetrics and Gynaecology, Jeroen Bosch Hospital, Den Bosch, The Netherlands for early-stage disease (FIGO stages I-II) is only 50\% and significantly drops to $10-20 \%$ for late-stage disease (FIGO stages III-IV) [2, 3].

Due to its rarity, no prospective trials on treatment are available. Therefore, there is a constant need for evaluation of the results of care [4]. A large amount of retrospective research has been published in the past few years, but there is

5 Department of Obstetrics and Gynaecology, Amphia Hospital, Breda, the Netherlands

6 Department of Surgery, Saint Francis Mission Hospital, Katete, Zambia

7 Department of Obstetrics and Gynecology, Harapan Kita Women and Children Hospital, University of Indonesia, Jakarta, Indonesia 
still lack of evidence on the best treatment options for UCS patients $[2,5,6]$. The standard of care in early-stage disease is surgery consisting of hysterectomy with or without bilateral salpingo-oophorectomy [3]. For late-stage disease, cytoreductive surgery has shown to increase OS [3, 7]. Concerning adjuvant therapy, there is ongoing discussion whether it improves survival and until now no evidence-based treatment strategy is established $[8,9]$. This retrospective study evaluates the clinical presentation, treatment and survival of Caucasian patients diagnosed with UCS in the Comprehensive Cancer Network south region in the Netherlands.

\section{Materials and Methods}

\section{Study Design and Population}

This study has a multicenter retrospective cohort design. Medical ethical approval was granted from the medical ethical committee. We collected data on treatment of UCS patients who were diagnosed between 2004 and 2014. Patients were identified through our online database registration. Data were retrospectively retrieved from our online database registration and completed by reviewing the patients' medical records. We abstracted patient characteristics (age at diagnose, FIGO stage, tumor size, menopausal status, symptoms, relapse location) and treatment data (surgery with or without adjuvant therapy and chemotherapy or radiotherapy only). For patients who underwent surgery, the type of surgery was documented (hysterectomy alone, hysterectomy and bilateral salpingo-oophorectomy (HTBSO), HT-BSO with pelvic and/or para-aortic lymphadenectomy and debulking surgery). Patients received follow-up according to national guidelines. Survival and disease-free survival were calculated. Disease recurrences were categorized into pelvic and distant (outside pelvic).

\section{Statistical Analysis}

Data were coded and entered into a database and were analyzed with SPSS version 23. Mean and standard deviations (SD) are reported for continuous variables and frequency counts and percentage for categorical variables. Survival curves were generated using the Kaplan-Meier method. A p-value less than 0.05 was considered statistically significant.

\section{Results}

\section{Patient Demographics}

During the study period, 62 UCS patients were identified. The mean age at time of diagnosis was 69.2 years (range
45-95 years). Six patients were excluded, four patients because they did not receive any treatment due to their performance status and two patients because of incomplete data. The median follow-up period was 70 months (range 36-114). Abnormal vaginal bleeding was the most frequent presenting symptom $(80.4 \%)$. Assuming that patients older than 52 years were postmenopausal, 52 patients were postmenopausal $(92.9 \%)$. In this study, recurrence occurred between 1 and 37 months after primary treatment. Thirtyseven patients $(66.1 \%)$ have no recurrence, and 19 patients (33.9\%) have recurrence in difference organs. For all recurrence patients, five patients were treated with surgery alone $(26.3 \%)$, five patients had surgery combined with adjuvant chemotherapy $(26.3 \%)$, eight patients had surgery combined with adjuvant radiotherapy (42.1\%), and one patient had chemotherapy alone (5.3\%). In 26 patients, the initial tumor size was known and the median size of the tumor was $6 \mathrm{~cm}$ (IQR 2.1-13 cm). According to the FIGO classification, $32(57.1 \%)$ patients had FIGO early-stage (III) disease and 24 (42.9\%) patients had FIGO late-stage (III-IV) disease. Patients' characteristics are shown in Table 1.

\section{Treatment Modalities}

Of the 56 included patients, all received at least one treatment modality. Fifty-three patients (94.6\%) were treated surgically. Nineteen patients $(33.9 \%)$ were treated with surgery only, and 34 patients $(60.7 \%)$ also received postoperative adjuvant therapy. Fifteen (26.8\%) patients had surgery combined with adjuvant chemotherapy, 19 (33.9\%) patients had surgery combined with adjuvant radiotherapy, and three patients $(5.4 \%)$ received only chemotherapy without any other treatment. Hysterectomy with bilateral salpingo-oophorectomy (BSO) was the most performed surgical procedure in 30 cases $(53.6 \%)$ (Table 2). Of the patients with FIGO early-stage (I-II) disease, $46.9 \%$ received surgery alone, whereas $9.4 \%$ received adjuvant chemotherapy and $43.8 \%$ adjuvant radiotherapy. Of the patients with FIGO late-stage (III-IV) disease, $16.7 \%$ received surgery alone, whereas $50 \%$ received adjuvant chemotherapy, $20.8 \%$ adjuvant radiotherapy and $12.5 \%$ chemotherapy alone.

\section{Survival and Recurrence}

The overall five-year survival rate was 55\% (95\% CI 42.7-68.3\%). A significant difference in 5-year OS was seen between patients with FIGO stage I-II (early-stage) disease (75\%; 95\% CI 59.5-91.5\%) and FIGO stage III-IV (late-stage) disease $\quad(25 \% ; \quad 95 \% \quad$ CI $\quad 13.5-36.7 \%)$ 
Table 1 Characteristics and symptoms of patients with uterine carcinosarcoma

\begin{tabular}{ll}
\hline Median age (years) (IQR) & $69.2(45-95)$ \\
Symptoms & \\
Vaginal bleeding & $45(80.4 \%)$ \\
Abdominal enlargement & $2(3.6 \%)$ \\
Pain & $7(12.5 \%)$ \\
Urinary retention & $1(1.8 \%)$ \\
Uterine prolapse & $1(1.8 \%)$ \\
Postmenopausal & \\
Yes & $52(92.9 \%)$ \\
No & $4(7.1 \%)$ \\
Type of recurrence & \\
No recurrence & $37(66.1 \%)$ \\
Lung & $4(7.1 \%)$ \\
Abdominal & $4(7.1 \%)$ \\
Local & $7(12.5 \%)$ \\
Other & $4(7.1 \%)$ \\
Median tumor size (IQR) & $6 \mathrm{~cm} \mathrm{(2.1-13} \mathrm{cm)}$ \\
FIGO stage & \\
I & $31(67 \%)$ \\
II & $1(4 \%)$ \\
III & $14(25.1 \%)$ \\
IV & $10(13 \%)$ \\
\hline
\end{tabular}

Table 2 Treatment modalities of uterine carcinosarcoma

\begin{tabular}{ll}
\hline Surgery & \\
Surgery without adjuvant & $19(33.9 \%)$ \\
Surgery with adjuvant & $34(60.7 \%)$ \\
$\quad$ Radiotherapy (RT) & $19(33.9 \%)$ \\
$\quad$ Chemotherapy (CT) & $15(26.8 \%)$ \\
Non-surgical treatment (CT) & $3(5.4 \%)$ \\
Type of surgery & \\
Hysterectomy & $4(7.1 \%)$ \\
Hysterectomy + BSO & $30(53.6 \%)$ \\
Hysterectomy + BSO + lymphadenectomy & $8(14.3 \%)$ \\
Debulking & $11(19.6 \%)$ \\
Surgical route & \\
Non-surgical treatment & $3(5.4 \%)$ \\
Laparotomy & $50(89.2 \%)$ \\
Laparoscopy & $2(3.6 \%)$ \\
Vaginal & $1(1.8 \%)$ \\
\hline
\end{tabular}

$R T$ radiotherapy, $C T$ chemotherapy, $H T$ hormone therapy

( $p=0.000)$. The median overall survival (OS) for patients with FIGO stage I-II was 47.9 months (IQR 24.7-79.8 months) and for patients with FIGO stage III-IV was 12.4 months (IQR 4.4-31.9 months) (Fig. 1a).

The five-year disease-free survival (DFS) for all patients was 52\% (95\% CI 38.6-65.4\%). A significant difference was seen in DFS of patients diagnosed with FIGO I-II and FIGO III-IV disease [72.4\%; 95\% CI 55.4-89.5\%, and 20.6\%; 95\% CI 8.9-32.4\%, respectively $(p=0.000)$ ]. The median disease-free survival (DFS) for patients with FIGO stages III was 47 months (IQR 17.5-72 months) and FIGO stages III-IV was 8.5 months (IQR 2.5-8.5 months) (Fig. 1b).

No significant difference in five-year OS between patients based on type of treatment was observed: surgery alone $(71 \%$; 95\% CI 48-93\%), surgery with adjuvant chemotherapy (32\%; 95\% CI 15-48\%), surgery with adjuvant radiotherapy $(47 \%$; $95 \%$ CI $32-62 \%)$ and chemotherapy alone (20\%; 95\% CI 0-42\%) (log-rank $p=0.160$ ) (Fig. 2a). For recurrence rate, there was also no significant difference detected: surgery alone $(87 \%$; $95 \%$ CI $65-109 \%$ ), surgery with adjuvant chemotherapy (48\%; 95\% CI 25-72\%), surgery with adjuvant radiotherapy (56\%; 95\% CI 39-74\%) and chemotherapy alone (29\%; 95\% CI 29-29) (log-rank $p=0.651)$ (Fig. 2b).

Since difference in survival might be explained by tumor stage rather than treatment modality, we also calculated five-year overall survival (OS) based on type of treatment and FIGO stage. Again, no significant difference was seen in the subgroup analysis of early-stage disease: surgery alone, surgery with adjuvant chemotherapy and surgery with adjuvant radiotherapy (log-rank $p=0.226$ ) (Fig. 3a), and in that of late-stage disease: surgery alone, surgery with adjuvant chemotherapy, surgery with adjuvant radiotherapy and chemotherapy alone (log-rank $p=0.706)$ (Fig. 3b).

\section{Discussion}

UCS is highly aggressive and has a poor prognosis [10] as also shown in our cohort of patients. Currently, there is no clear guideline for therapeutic management of carcinosarcoma. Optimal treatment strategies are still not established for both surgery and adjuvant treatment [7, 8], although surgery is still considered the corner stone of treatment of this disease. Due to limited data and only few prospective trials on treatment of this disease, this issue has not been clarified [11]. Consequently, the need for individual cancer centers to report study data for clinical outcome of this rare disease remains.

There are several known risk factors for UCS including postmenopausal age, tamoxifen use and previous irradiation [11]. $92.9 \%$ of the women in our study were postmenopausal, but none had a history of previous pelvic irradiation or tamoxifen use. Previously reported mean of age at diagnosis is 62 years [9], which is in line with our results (69.2 years).

As shown by others, we demonstrated a better survival for FIGO early-stage disease versus late-stage disease 
Fig. 1 Kaplan-Meier curves: a overall survival of patients with FIGO stages I-II versus FIGO stages III-IV disease (log-rank $p=<0.001$ ), b disease-free survival of patients with FIGO stages I-II versus FIGO stages III-IV (logrank $p=<0.001$ )

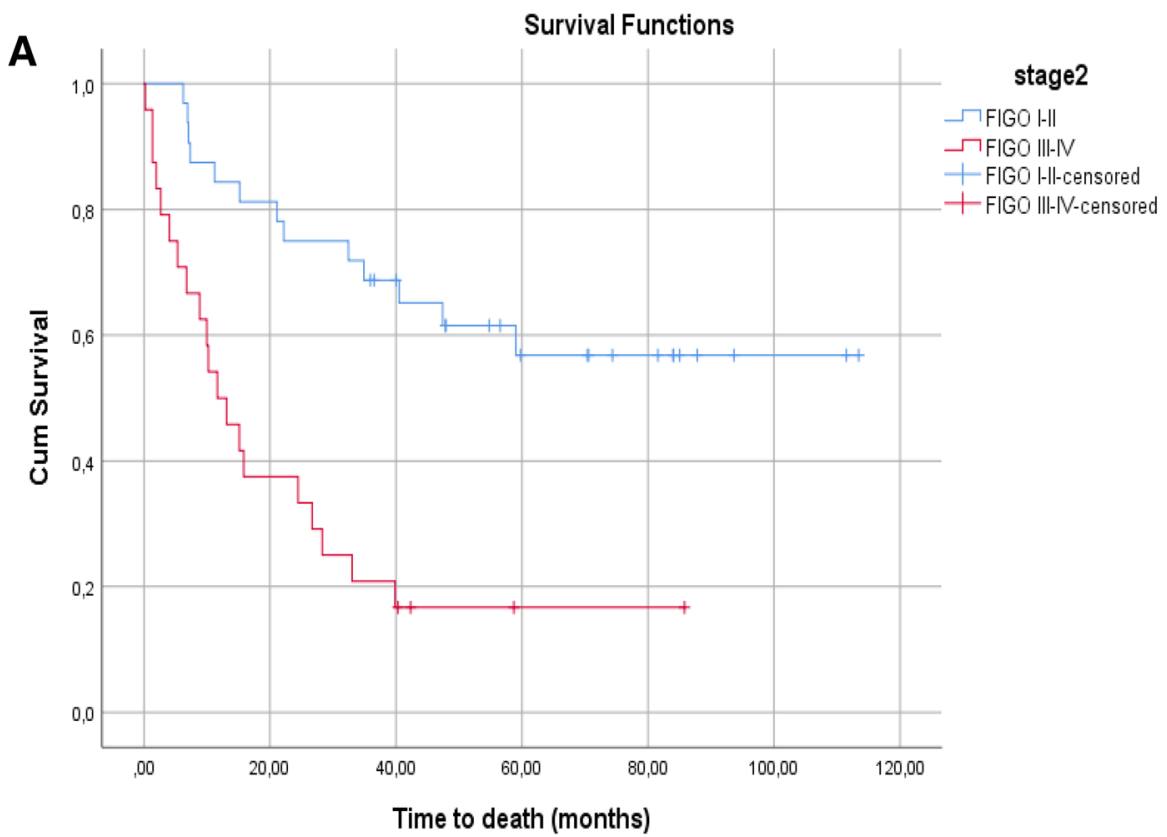

B

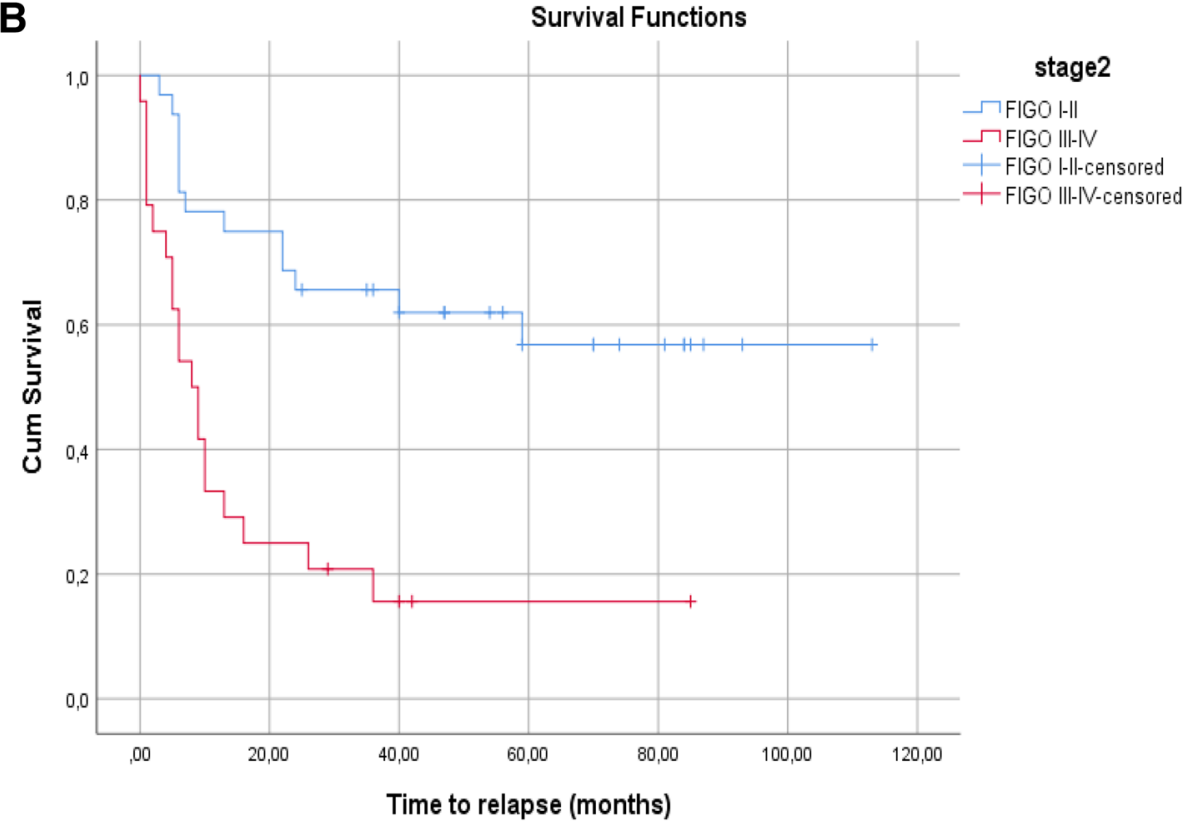

(75\% vs $25 \%$ ). This was also shown in a previous study by the Japanese Gynecology Oncology Group on 486 patients, of whom $256(53 \%)$ had FIGO early-stage disease and 230 (47\%) had FIGO late-stage disease [12]. The estimated 5 -year survival for patients with USC is poor ranging from 33 to $39 \%$ [4, 13]. In our study, 5-year overall survival was slightly higher $55 \%$. The reason might be that $57 \%$ of our patients had FIGO early-stage disease.

As also shown in other studies Wolfson et al. [14] and Reed et al. [15], no significant difference in survival was observed in our study based on (adjuvant) treatment in all stages of disease. However, a previous study by Manzerova et al., one of the largest observational cohort studies for UCS in the literature, included 2342 patients from the Surveillance, Epidemiology and End Results (SEER) database who were treated with surgery with or without adjuvant radiation therapy (aRT). OS was significantly longer in adjuvant RT arm compared with the observational arm $(42 \%$ vs $22 \%)(p=0.0001)$ [16]. Also, Zwallen et al. retrospectively studied 124 patients with UCS; postoperative pelvic adjuvant external beam radiotherapy (EBRT) was given to 105 patients and additional or 
Fig. 2 Kaplan-Meier curves: a overall survival of UCS patients based on type of treatment in all stages (log-rank $p=0.160)$, $\mathbf{b}$ disease-free survival (log-rank $p=0.615)$
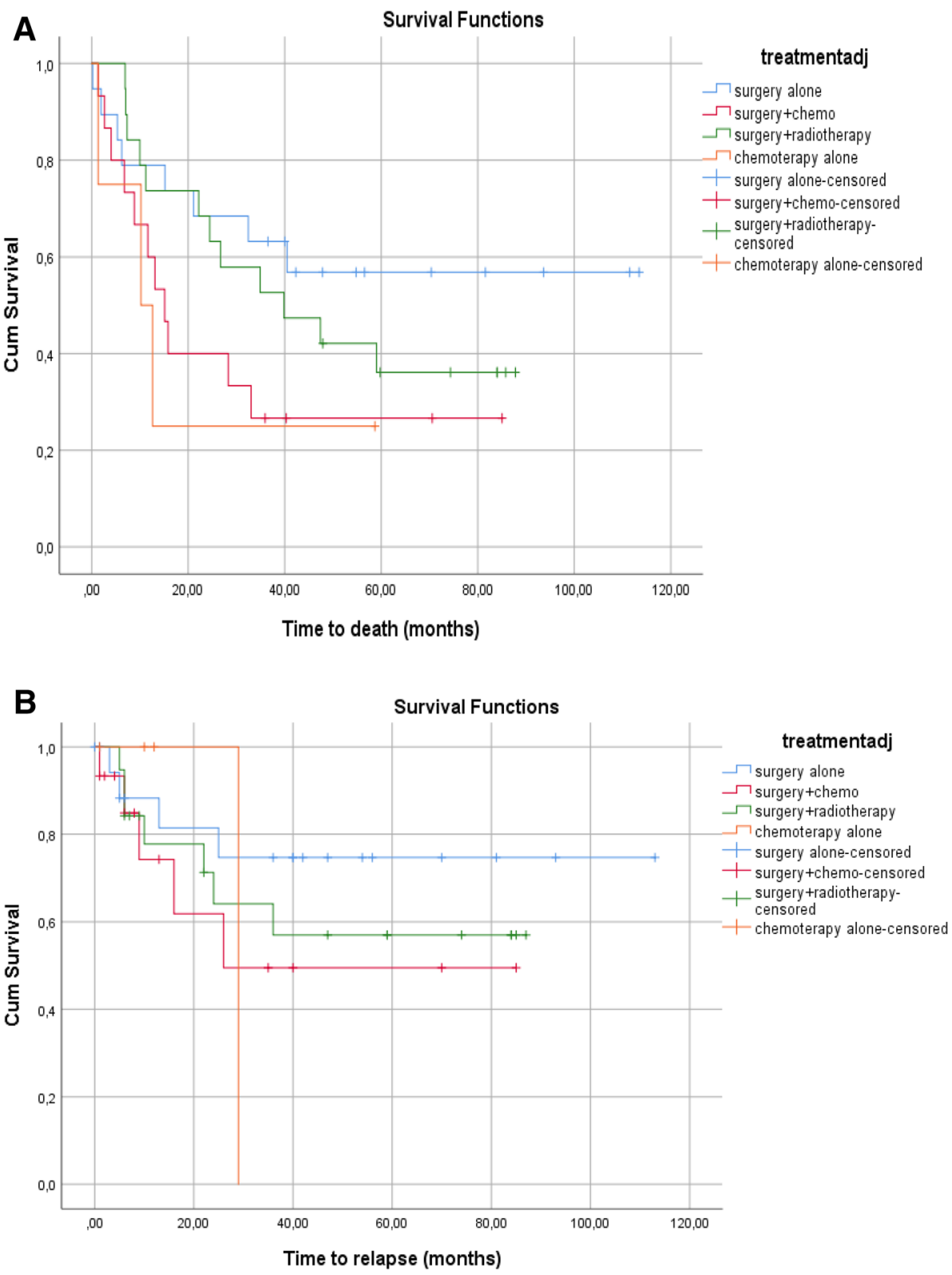

exclusive brachytherapy (BT) to 92 patients after total hysterectomy. This study showed that EBRT and BT dose, early stage and younger age resulted in improved OS, DFS and locoregional control. This result was also found in the EORTC 55874, and our study that showed stage of disease is one of the most important predictors of survival and that postoperative radiotherapy improved locoregional control. The same result was also found in a recently published large cohort study involving 1581 women by Patel et al., which showed that younger age at diagnosis, race and FIGO stage were found to be significant predictors of both OS and DFS. This study also showed a nonsignificant role for adjuvant radiotherapy (EBRT $\pm \mathrm{BT}$ vs BT alone) in UCS patients. BT alone did not show to have worse OS than EBRT, suggesting that vaginal BT may be an effective alternative to EBRT for adjuvant therapy for UCS. Again, this finding is not clear enough and should be interpreted with caution, as different confounders could influence the analysis and the conclusions from this study, which is limited due to its non-randomized design [17]. In contrast, the latter study showed that postoperative RT resulted in a 5 -year OS of $51.6 \%$ (95\% CI 35-73\%) and cancer-specific survival (CSS) of $58.6 \%$ (95\% CI 38-74\%), showing one of the highest OS and CSS in the literature to date. However, these findings should also be interpreted with caution due to small cohort size and potential selection bias [18]. In summary, the effect of postoperative adjuvant RT of UCS remains controversial, some studies only report local 
Fig. 3 Kaplan-Meier curves: a overall survival of UCS patients based on type of treatment in early-stage disease (log-rank $p=0.226$ ), b in latestage disease (log-rank $p=0.706$ )
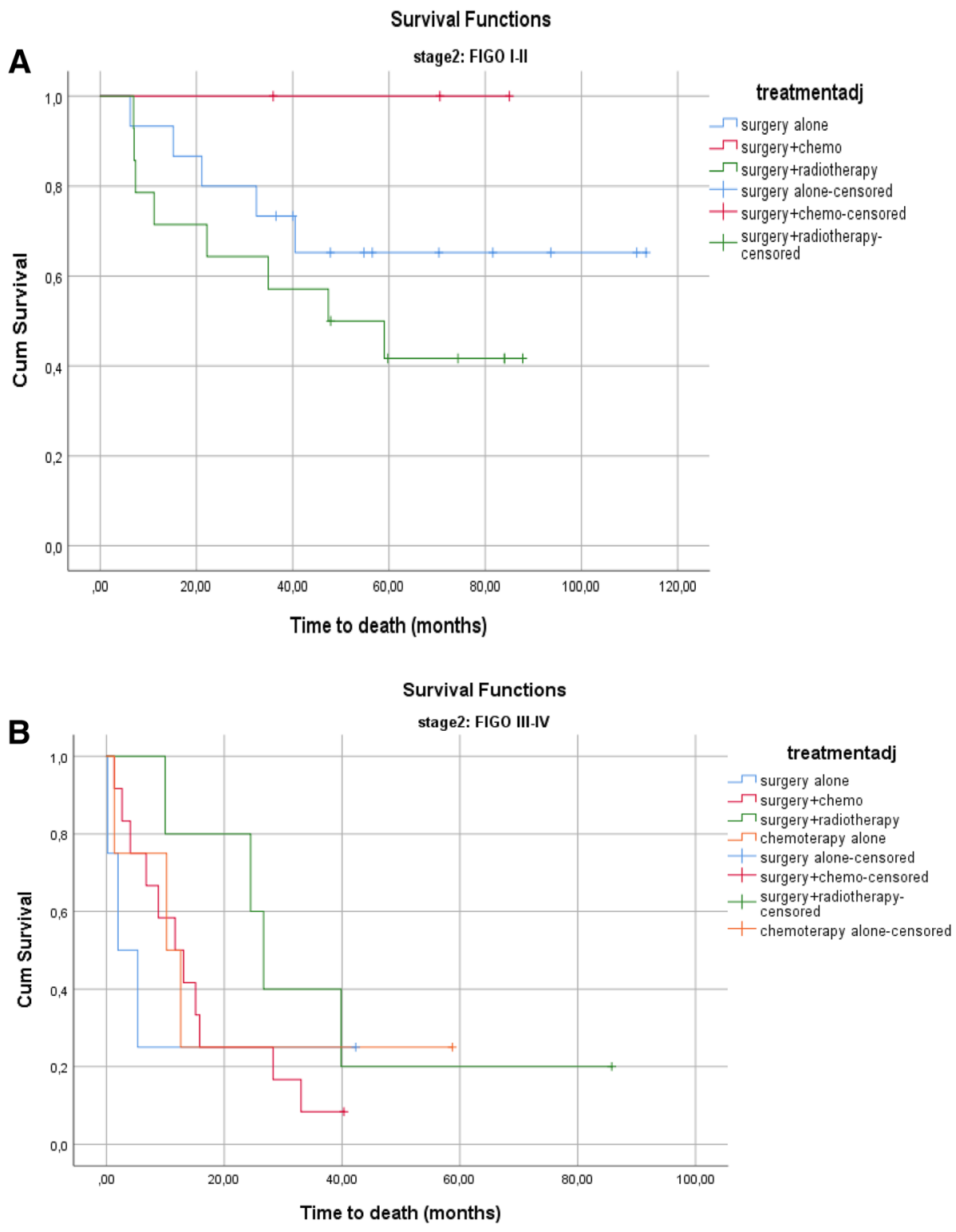

control without a survival benefit, and other studies report an improvement in survival with adjuvant RT.

Only two randomized trials have been completed with respect to adjuvant therapy in UCS. Firstly, a prospective randomized control trial phase III (GOG Protocol 150) was designed to compare whole-abdominal irradiation (WAI) vs therapy combination cisplatin and ifosfamide along with mesna (CIM). This study concluded that recurrence rates and survival are not altered by the addition of adjuvant therapy in patients with UCS, although there were a significant increase in late adverse events in adjuvant RT (WAI) and increased vaginal recurrences in the chemotherapy group. Five-year probability of relapse was $58 \%$ vs $52 \%$ for the WAI vs CIM, and the estimated recurrence rate in CIM was $29 \%$ lower than the WAI patients. Secondly, a trial to evaluate adjuvant radiotherapy was conducted by the European Organization for Research and Treatment of Cancer (EORTC 55874) Gynecological Cancer Group Study, in which 224 patients were included (91 patients with early-stage UCS disease) and randomized after primary surgery to either observation arm or pelvic radiation (EBRT) arm. This study revealed improved locoregional control in adjuvant radiotherapy (24\% vs $47 \%$ ) compared with the observational group, especially in early-stage disease (FIGO stages I-II). However, no difference in either overall or disease-free survival was demonstrated [15]. This observation might have been caused by the high risk of distant metastases, even in early stage $32 \%$ of patients had extra-uterine disease spread [19]. In our cohort study, 33.9\% of all patients received adjuvant radiotherapy. Forty-two percent of patients developed a recurrence during the time of this study, and only $12.5 \%$ of 
these patients had a local recurrence. So, our results are in line with the above-mentioned studies. Adjuvant RT improved locoregional control, but the majority of patients in our cohort treated with irradiation had recurrences at distant sites. This indicates that patients require also a systemic agent, which reduces distant site recurrences.

A retrospective study from Otsuki et al. on 45 UCS patients that received postoperative chemotherapy showed that both the FIGO stage $(p=0.0034)$ and postoperative combination chemotherapy $(p=0.0049)$ were significantly predictors of survival for all FIGO-stage UCS patients who underwent hysterectomy and bilateral salpingo-oophorectomy [20]. These results are similar to those of the Homesley et al. and Sutton et al. trial that showed the risk of death and DFS was lower among women who received combination chemotherapy compared with single adjuvant chemotherapy (ifosfamide alone) in late-stage and recurrent disease. However, it remains unknown whether postoperative adjuvant chemotherapy really improves prognosis for FIGO stage I-II patients [21, 22].

Our study has the limitations accompanied by a retrospective cohort study, and the number of patients is low. Also due to the small sample size and lack of data, analysis could only be made for early-stage and late-stage disease and not for type of treatment. The strength of our study is the completeness of the cohort of patients with this rare disease.

In conclusion, in this study UCS with both FIGO stages I-II and III-IV has a dismal prognosis, although late-stage disease has the worse prognosis. The addition of adjuvant treatment did not seem to increase survival.

Acknowledgements This research did not receive any specific grant from funding agencies in the public, commercial or not-for-profit sectors.

\section{Compliance with Ethical Standards}

Conflict of interest There is no conflict of interest relevant to this article.

Open Access This article is licensed under a Creative Commons Attribution 4.0 International License, which permits use, sharing, adaptation, distribution and reproduction in any medium or format, as long as you give appropriate credit to the original author(s) and the source, provide a link to the Creative Commons licence, and indicate if changes were made. The images or other third party material in this article are included in the article's Creative Commons licence, unless indicated otherwise in a credit line to the material. If material is not included in the article's Creative Commons licence and your intended use is not permitted by statutory regulation or exceeds the permitted use, you will need to obtain permission directly from the copyright holder. To view a copy of this licence, visit http://creativecommons. org/licenses/by/4.0/.

\section{References}

1. Guttmann DM, Li H, Sevak P, Grover S, Jacobson G, Feldman A, et al. The impact of adjuvant therapy on survival and recurrence patterns in women with early-stage uterine carcinosarcoma: a multi-institutional study. Int J Gynecol Cancer. 2016;26(1):141-8

2. Cantrell LA, Havrilesky L, Moore DT, O’Malley D, Liotta M, Secord AA, et al. A multi-institutional cohort study of adjuvant therapy in stage I-II uterine carcinosarcoma. Gynecol Oncol. 2012;127(1):22-6.

3. Denschlag D, Thiel FC, Ackermann S, Harter P, Juhasz-Boess I, Mallmann P, et al. Sarcoma of the uterus Guideline of the DGGG (S2k-Level, AWMF Registry No. 015/074, August 2015). Geburtshilfe Frauenheilkd. 2015;75(10):1028-42.

4. Cantrell LA, Blank SV, Duska LR. Uterine carcinosarcoma: a review of the literature. Gynecol Oncol. 2015;137(3):581-8.

5. Dickson EL, Vogel RI, Gehrig PA, Pierce S, Havrilesky L, Secord AA, et al. A multi-institutional study of outcomes in stage I-III uterine carcinosarcoma. Gynecol Oncol. 2015;139(2):275-82.

6. Gokce ZK, Turan T, Karalok A, Tasci T, Ureyen I, Ozkaya E, et al. Clinical outcomes of uterine carcinosarcoma: results of 94 patients. Int J Gynecol Cancer. 2015;25(2):279-87.

7. Wallwiener C, Hartkopf A, Kommoss S, Joachim C, Wallwiener $\mathrm{M}$, Taran FA, et al. Clinical characteristics, surgical management and adjuvant therapy of patients with uterine carcinosarcoma: a retrospective case series. Geburtshilfe Frauenheilkd. 2016;76(2):188-93.

8. Menczer J. Review of recommended treatment of uterine carcinosarcoma. Curr Treat Options Oncol. 2015;16(11):53.

9. Gungorduk K, Ozdemir A, Ertas IE, Gokcu M, Telli E, Oge T, et al. Adjuvant treatment modalities, prognostic predictors and outcomes of uterine carcinosarcomas. Cancer Res Treat. 2015;47(2):282-9.

10. Artioli G, Wabersich J, Ludwig K, Gardiman MP, Borgato L, Garbin F. Rare uterine cancer: carcinosarcomas. Review from histology to treatment. Crit Rev Oncol Hematol. 2015;94(1):98-104.

11. Koh WJ, Greer BE, Abu-Rustum NR, Apte SM, Campos SM, Cho KR, et al. Uterine sarcoma, version 1.2016: featured updates to the NCCN guidelines. J Natl Compr Cancer Netw. 2015;13(11):1321-31.

12. Harano K, Hirakawa A, Yunokawa M, Nakamura T, Satoh T, Nishikawa $\mathrm{T}$, et al. Prognostic factors in patients with uterine carcinosarcoma: a multi-institutional retrospective study from the Japanese Gynecologic Oncology Group. Int $\mathrm{J}$ Clin Oncol. 2016;21(1):168-76.

13. Vorgias G, Fotiou S. The role of lymphadenectomy in uterine carcinosarcomas (malignant mixed mullerian tumours): a critical literature review. Arch Gynecol Obstet. 2010;282(6):659-64.

14. Wolfson AH, Brady MF, Rocereto T, Mannel RS, Lee YC, Futoran RJ, et al. A gynecologic oncology group randomized phase III trial of whole abdominal irradiation (WAI) vs. cisplatin-ifosfamide and mesna (CIM) as post-surgical therapy in stage I-IV carcinosarcoma (CS) of the uterus. Gynecol Oncol. 2007;107(2):177-85.

15. Reed NS, Mangioni C, Malmstrom H, Scarfone G, Poveda A, Pecorelli S, et al. Phase III randomised study to evaluate the role of adjuvant pelvic radiotherapy in the treatment of uterine sarcomas stages I and II: an European Organisation for Research and Treatment of Cancer Gynaecological Cancer Group Study (protocol 55874). Eur J Cancer. 2008;44(6):808-18.

16. Manzerova J, Sison CP, Gupta D, Holcomb K, Caputo TA, Parashar B, et al. Adjuvant radiation therapy in uterine 
carcinosarcoma: a population-based analysis of patient demographic and clinical characteristics, patterns of care and outcomes. Gynecol Oncol. 2016;141(2):225-30.

17. Patel N, Hegarty SE, Cantrell LA, Mishra MV, Showalter TN. Evaluation of brachytherapy and external beam radiation therapy for early stage, node-negative uterine carcinosarcoma. Brachytherapy. 2015;14(5):606-12.

18. Zwahlen DR, Schick U, Bolukbasi Y, Thariat J, Abdah-Bortnyak $\mathrm{R}$, Kuten A, et al. Outcome and predictive factors in uterine carcinosarcoma using postoperative radiotherapy: a rare cancer network study. Rare Tumors. 2016;8(2):6052.

19. Inthasorn P, Carter J, Valmadre S, Beale P, Russell P, Dalrymple C. Analysis of clinicopathologic factors in malignant mixed Müllerian tumors of the uterine corpus. Int $\mathrm{J}$ Gynecol Cancer. 2002;12(4):348-53.

20. Otsuki A, Otsuki T, Tokunaga H, Niikura H, Nagase S, Sugiyama $\mathrm{T}$, et al. Evaluation of postoperative chemotherapy in patients with uterine carcinosarcoma: a retrospective survey of the Tohoku Gynecologic Cancer Unit. Int $\mathbf{J}$ Clin Oncol. 2015;20(3):574-8.

21. Sutton G, Brunetto VL, Kilgore L, Soper JT, McGehee R, Olt G, et al. A phase III trial of ifosfamide with or without cisplatin in carcinosarcoma of the uterus: a Gynecologic Oncology Group Study. Gynecol Oncol. 2000;79(2):147-53.

22. Homesley HD, Filiaci V, Markman M, Bitterman P, Eaton L, Kilgore LC, et al. Phase III trial of ifosfamide with or without paclitaxel in advanced uterine carcinosarcoma: a Gynecologic Oncology Group Study. J Clin Oncol. 2007;25(5):526-31.

Publisher's Note Springer Nature remains neutral with regard to jurisdictional claims in published maps and institutional affiliations. 